\title{
RECURRENCE RELATIONS, DYNKIN DIAGRAMS AND PLÜCKER FORMULAE
}

\author{
JOHN M. BURNS \\ Department of Mathematics, National University of Ireland, Galway, Ireland \\ and MICHAEL J. CLANCY \\ School of Mathematical Sciences, Dublin City University, Dublin 9, Ireland
}

(Received 27 January, 2006; revised 27 September, 2006)

\begin{abstract}
We prove a generalisation of an observation of N. Iwahori concerning the coefficients of the extended Dynkin diagram of a complex simple Lie algebra. We relate the combinatorics of these coefficients to the orders of finite groups that act discontinuously on the Riemann sphere and to the Plücker formulae.
\end{abstract}

2000 Mathematics Subject Classification. 17B20.

1. Introduction. In this note we prove a generalisation of the following observation of $\mathbf{N}$. Iwahori that appears in [5]. Let $\mathbf{g}$ be a complex simple Lie algebra with extended Dynkin diagram $\tilde{\triangle}_{\mathbf{g}}$. The vertex coefficients of any simple path in $\tilde{\triangle}_{\mathbf{g}}$, starting at a pendant vertex and ending at the first vertex with a branch or multiple connection, form an arithmetic progression. We show how this observation leads to a simple combinatorial derivation of the well-known correspondence between the Dynkin diagrams of simply laced complex simple Lie algebras (i.e. types $A, D$ or $E$ ) and the finite subgroups of $S U(2)$. Finally we relate our results to the Plücker formulae as generalised in [1]. The first author would like to thank the Centre de Mathématiques et d'Informatique, Université de Provence, Aix-Marseille I, where some of this work was carried out, for its hospitality.

2. The observation. All basic facts and definitions used can be found in [2] or [4]. Throughout, we fix a Cartan subalgebra $\mathbf{h}$ of $\mathbf{g}$ and a fundamental Weyl chamber in $\mathbf{h}$. We denote the roots and positive roots by $\Re$ and $\mathfrak{R}^{+}$respectively, and the positive simple roots by $\left\{\alpha_{1}, \ldots, \alpha_{r}\right\}$, where $r$ is the rank of $\mathbf{g}$. The corresponding fundamental weights $\left\{\omega_{1}, \ldots, \omega_{r}\right\}$ are defined by the conditions $\left\langle\omega_{i}, 2 \alpha_{j}\right\rangle:=\left\langle\alpha_{j}, \alpha_{j}\right\rangle \delta_{i j} \forall i, j$, where $\langle, \quad\rangle$ is an invariant innerproduct, normalized so that the highest root $\tilde{\alpha}$ has length squared equal to two. For $\alpha, \beta \in \Re$ we define (integers) $n(\alpha, \beta)=\frac{2\langle\alpha, \beta\rangle}{\langle\alpha, \alpha\rangle}$, and the Cartan matrix $C(\mathbf{g})$ has entries $c_{i j}=n\left(\alpha_{i}, \alpha_{j}\right)$. The Dynkin diagram $\triangle_{\mathbf{g}}$ is the (multi) graph with $r$ vertices (labelled by the positive simple roots), and $c_{i j} c_{j i}$ edges joining $\alpha_{i}$ to $\alpha_{j}$. We observe that when $\mathbf{g}$ is simply laced (i.e. all roots have the same length), $\triangle_{\mathbf{g}}$ has adjacency matrix $A(\mathbf{g})=2 I_{r}-C(\mathbf{g})$, where $I_{r}$ is the $r \times r$ identity matrix. The extended Dynkin diagram $\tilde{\triangle}_{\mathbf{g}}$ is the graph constructed from $\triangle_{\mathbf{g}}$ by adding a new vertex $\alpha_{0}=-\tilde{\alpha}$ and joining it to any vertex $\alpha_{i}$ by (the old rule of) $n\left(\alpha_{i}, \tilde{\alpha}\right) \cdot n\left(\tilde{\alpha}, \alpha_{i}\right)$ edges. We then write the coefficient $m_{i}$ over the vertex $\alpha_{i}$ and $m_{0}=1$ over $\alpha_{0}$, where $\tilde{\alpha}=\sum_{i=1}^{r} m_{i} \alpha_{i}$. Similarly the extended Dynkin diagram $\tilde{\triangle}_{\omega_{j}}$ of a fundamental irreducible representation of $\mathbf{g}$ 
with highest weight $\omega_{j}$ has vertices the positive simple roots (joined by the old rule) and an additional vertex $\alpha_{0}$ with $\delta_{i j}$ edges joining it to any vertex $\alpha_{i}$. We then write the coefficient $d_{i}^{j}$ over the vertex $\alpha_{i}$ and $d_{0}^{j}=1$ over $\alpha_{0}$, where $\omega_{j}=\sum_{i=1}^{r} d_{i}^{j} \alpha_{i}$. Using this notation we have the following generalisation of $\mathrm{N}$. Iwahori's observation.

Proposition 2.1. The vertex coefficients $d_{i}^{j}$ of any simple path in $\tilde{\triangle}_{\omega_{i}}$, starting at a pendant vertex and ending at the first vertex with a branch or multiple connection, form an arithmetic progression. In particular the vertex coefficients $m_{i}$ of any such simple path in $\tilde{\triangle}_{\mathbf{g}}$, form an arithmetic progression.

Proof. Since $\alpha_{i}=\sum_{k=1}^{r} c_{k i} \omega_{k}$ we have $\alpha_{i}=2 \omega_{i}+\sum_{k \in N(i)} c_{k i} \omega_{k}$, where $k \in N(i)$ if and only if $\alpha_{k}$ is adjacent to $\alpha_{i}$ in the Dynkin diagram. Taking inner-products with $\omega_{j}$ we obtain

$$
\delta_{i j}=2 d_{i}^{j}+\sum_{k \in N(i)} d_{k}^{j} c_{k i} \frac{\left\langle\alpha_{k}, \alpha_{k}\right\rangle}{\left\langle\alpha_{i}, \alpha_{i}\right\rangle}
$$

and therefore

$$
\delta_{i j}=2 d_{i}^{j}+\sum_{k \in N(i)} c_{i k} d_{k}^{j} .
$$

For the sake of clarity we first consider the simply laced cases, so that the off diagonal entries of the Cartan matrix and the adjacency matrix satisfy $c_{j i}=c_{i j}=-a_{i j}=-1$ for adjacent vertices $i$ and $j$, and are zero otherwise. Our equations then become $2 d_{i}^{j}=$ $\sum_{k \in N(i)} d_{k}^{j}+\delta_{i j}$. When $\alpha_{i}$ is neither a pendant, nor a branch vertex, our equations can be written as $2 d_{i}^{j}=d_{i^{-}}^{j}+d_{i^{+}}^{j}+\delta_{i j}$, since in this case $N(i)$ contains only two elements $i^{-}$and $i^{+}$, with the minus sign attached to the node that is closer to the pendant vertex. We see therefore that $d_{i^{+}}^{j}-d_{i}^{j}=d_{i}^{j}-d_{i^{-}}^{j}-\delta_{i j}$. Thus for $i \neq j$ the vertex coefficients $d_{i}^{j}$ of such a path form an arithmetic progression. In order to evaluate the common difference we note that when $\alpha_{i}, \quad i \neq j$ is the pendant vertex we have $2 d_{i}^{j}=d_{i^{+}}^{j}$ and therefore $d_{i^{+}}^{j}-d_{i}^{j}=d_{i}^{j}$. When $i=j$ we may assume that $\alpha_{i}$ is a pendant vertex, since otherwise it is a branch node of $\tilde{\triangle}_{\omega_{j}}$ and the result is trivially true. Now $\alpha_{i^{+}}$has two neighbours, $\delta_{i^{+} j} \neq 1$, and the argument is as above, except the common difference is $d_{j}^{j}-1$. In the non-simply laced cases, we observe that when solving the recurrence relations $2 d_{i}^{j}=\sum_{k \in N(i)} c_{i k} d_{k}^{j}+\delta_{i j}$, we will only encounter a $c_{i k}=-2$ or -3 in solving beyond a vertex with a multiple edge, and therefore the simply laced argument applies up to such a vertex. To prove the final statement concerning $\tilde{\triangle}_{\mathbf{g}}$, we observe that when $\mathbf{g}$ is not of type $A_{r}, \tilde{\alpha}=f \omega_{j}$ for some $f \in \mathbb{Z}$, and when $\mathbf{g}$ is of type $A_{r}, \tilde{\triangle}_{\mathbf{g}}$ has no pendant vertex. To see this recall [2] that $\frac{2\langle\alpha, \tilde{\alpha}\rangle}{\langle\tilde{\alpha}, \tilde{\alpha}\rangle}=0$ or 1 for $\alpha$ positive unless $\alpha=\tilde{\alpha}$, and for non-proportional roots $\alpha$ and $\beta, \alpha+\beta$ is a root when $n(\alpha, \beta)<0$. If there are two simple roots $\alpha_{i}$ and $\alpha_{j}$ that are not orthogonal to $\tilde{\alpha}$, then $\alpha_{i}+\alpha+\alpha_{j}$ is a root where $\alpha$ is the sum of the intervening simple roots in any simple path in $\triangle_{\mathbf{g}}$ joining $\alpha_{i}$ to $\alpha_{j}$, and must therefore be $\tilde{\alpha}$. This means that $i=1, j=r, \tilde{\alpha}=\omega_{1}+\omega_{r}$ and $\Re$ is of type $A$. Similarly at most two simple roots are non-orthogonal to $\tilde{\alpha}$, and therefore for all other root systems $\tilde{\alpha}$ is a multiple of $\omega_{j}$ for some $j$.

REMARK. In order to have a non vacuous statement about the vertex coefficients $m_{i}$ in $\tilde{\triangle}_{\mathbf{g}}$ when $\mathfrak{R}$ is of type $A$, the role of a pendant vertex should be played by $\alpha_{0}$. Then the equations $2 m_{j}=\sum_{k \in N(j)} m_{k}, j=0, \ldots, r$ imply that the $m_{i}$ form a trivial arithmetic progression, as they are constant. 
3. Groups acting discontinuously on $\mathbb{C P}^{1}$. In this section we use Proposition 2.1 to study the coefficients $a_{i}$ defined by $2 \rho:=\sum_{i=1}^{r} a_{i} \alpha_{i}$, where $2 \rho$ denotes the sum of the positive roots. We also recall the classification due to Klein ( see [3] ) of the subgroups $\Gamma$ of $\operatorname{PSL}(2, \mathbb{C})$ that act discontinuously on $\mathbb{C P}^{1}$. Solving the recurrence relations satisfied by the $a_{i}$ 's, we relate in an elementary way these subgroups and the Dynkin diagrams of type $A, D$ and $E$. In keeping with the last section, we will view the coefficients $a_{i}$ as a function defined on the Dynkin diagram $\triangle_{\mathbf{g}}$. We first consider this function restricted to those paths in $\triangle_{\mathbf{g}}$ of the form considered in Proposition 2.1.

LEMMA 3.1. The coefficients $a_{i_{l}}$ of any simple path in $\triangle_{\mathbf{g}}$, starting at a pendant vertex $\alpha_{i_{1}}$ and ending at the first vertex $\alpha_{i_{t}}$ with a branch or multiple connection satisfy

$$
a_{i_{t}}=t a_{i_{1}}-t(t-1)
$$

and therefore

$$
a_{i_{t-1}}=\frac{t-1}{t}\left(a_{i_{t}}+t\right) .
$$

Proof. We first observe that from the identity $\sum_{i=1}^{r} a_{i} \alpha_{i}:=2 \rho=2 \sum_{j=1}^{r} \omega_{j}$, we obtain that $a_{i}=2 \sum_{j=1}^{r} d_{i}^{j}$. From Proposition 2.1 we have that for any vertex $\alpha_{i_{l}}$ in such a path $2 d_{i_{l}}^{j}=d_{i_{l-1}}^{j}+d_{i_{l+1}}^{j}+\delta_{i_{l j}}$ and therefore $\sum_{j=1}^{r} 2 d_{i_{l}}^{j}=\sum_{j=1}^{r}\left(d_{i_{l-1}}^{j}+d_{i_{l+1}}^{j}\right)+1$. By the observation above, we obtain $2 a_{i_{l}}=a_{i_{l-1}}+a_{i_{l+1}}+2$, so that $a_{i_{l+1}}-a_{i_{l}}=a_{i_{l}}-a_{i_{l-1}}+2$. We may solve this recurrence relation by treating it as an arithmetic progression after compensating for the occurrence of the 2 at each step, to obtain $a_{i_{t}}=t a_{i_{1}}-\sum_{l=1}^{t-1} 2 l=$ $t a_{i_{1}}-t(t-1)$. The corresponding formula for $a_{i_{t-1}}$ yields

$$
\begin{aligned}
a_{i_{t-1}} & =(t-1) a_{i_{1}}-(t-1)[(t-1)-1] \\
& =(t-1)\left[a_{i_{1}}-(t-1)+1\right] \\
& =\frac{t-1}{t}\left[t a_{i_{1}}-t(t-1)\right]=\frac{t-1}{t}\left(a_{i_{1}}+t\right) .
\end{aligned}
$$

THEOREM 3.1. Let $\triangle_{\mathbf{g}}$ be the Dynkin diagram of a simply laced complex simple Lie algebra of rank $r$, and let $\alpha_{s}$ be a node of $\triangle_{\mathbf{g}}$ with maximal degree $k$ and distances $v_{1}-1, \ldots, v_{k}-1$ from the corresponding pendant vertices. Then $a_{s}$ and $v_{1}, \ldots, v_{k}$ satisfy the classifying diophantine equation for a Kleinian group $\Gamma$ of signature $\left(0 ; v_{1}, \ldots, v_{k}\right)$ acting on the sphere, namely:

$$
\sum_{j=1}^{k}\left(1-\frac{1}{v_{j}}\right)=2-\frac{(1+r)}{a_{s}} .
$$

In particular we have that $|\Gamma|=\frac{2 a_{s}}{r+1}$.

Proof. Let $\pi: \mathbb{C P}^{1}: \rightarrow \mathbb{C P}^{1} / \Gamma$ be the canonical projection, and let $\left\{x_{1}, \ldots, x_{k}\right\}$ denote the points in the quotient Riemann surface $S$ such that $\pi$ is branched at $\pi^{-1}\left(x_{j}\right)$ with ramification index $v_{j}$. The Riemann-Hurwitz theorem implies that $S$ has genus zero and counting orbit sizes we get the well known diophantine equation:

$$
\sum_{j=1}^{k}\left(1-\frac{1}{v_{j}}\right)=2-\frac{2}{|\Gamma|} .
$$

This equation yields the classical list of five non-trivial subgroup types. Since we may regard $P S L(2, \mathbb{C})$ as the conformal transformations of $S^{2}$ and $S O(3)$ as its maximal 
compact group of isometries, the finite subgroups of both agree up to conjugation. We will therefore list the five subgroup types as $C_{n}$ (the cyclic group of order $n$ ), $D_{2 n}$ (the dihedral group of order $2 n$ ), $A_{4}, S_{4}$, and $A_{5}$. On the other hand by Lemma 3.1, $a_{s}$ (for a Dynkin diagram of type $A, D$, or $E$ ) satisfies the $k$ equations

$$
a_{i_{j-1}}=\frac{v_{j}-1}{v_{j}}\left(a_{s}+v_{j}\right) \quad(1 \leq j \leq k)
$$

where $v_{1}, \ldots, v_{k}$ are the branch lengths. Summing these equations we obtain

$$
\sum_{j=1}^{k} a_{i_{j}-1}=a_{s}\left(\sum_{j=1}^{k} \frac{v_{j}-1}{v_{j}}\right)+\sum_{j=1}^{k}\left(v_{j}-1\right)=a_{s}\left(\sum_{j=1}^{k} \frac{v_{j}-1}{v_{j}}\right)+r-1 .
$$

In addition we have $2 a_{s}=\sum_{m \in N(s)} a_{m}+2$, so that $\sum_{j=1}^{k} a_{i_{v_{j}-1}}=2 a_{s}-2$ and we get that

$$
\left(\sum_{j=1}^{k}\left(1-\frac{1}{v_{j}}\right)-2\right) a_{s}=-(1+r)
$$

Comparing this equation with the Kleinian group equation we see that $|\Gamma|=\frac{2 a_{s}}{r+1}$. There is a minor difference between solving this equation to classify the simply laced Dynkin diagrams and solving the corresponding equation for the finite subgroups of $\operatorname{PSL}(2, \mathbb{C})$. This occurs in the case $k=2,\left(A_{r}\right)$ where we may not assume that $\frac{1}{v_{1}}, \frac{1}{v_{2}} \geq \frac{r+1}{2 a_{s}}$, so that unlike the group case where $\frac{1}{v_{1}}, \frac{1}{v_{2}} \geq \frac{1}{|\Gamma|}$ we do not have the solution $v_{1}=v_{2}$ forced on us. Instead we may have $1 \leq v_{1} \leq r-1$ corresponding to each of the possible choices of a maximal degree node $\alpha_{s}$. We then have

$$
\frac{1}{s}+\frac{1}{r+1-s}=\frac{1+r}{a_{s}} \text { and } a_{s}=s(r+1-s) .
$$

However, since

$$
\sum_{j=1}^{k}\left(1-\frac{1}{v_{j}}\right)=2-\frac{1+r}{a_{s}}
$$

we see that in order for $|\Gamma|=\frac{2 a_{s}}{r+1}$ to be an integer, we must have $r$ odd, and choose the middle vertex $\alpha_{s}, s=\frac{r+1}{2}$ as the node of maximal degree. Then, $|\Gamma|=2 \frac{(r+1)^{2}}{4} \frac{1}{r+1}=$ $\frac{r+1}{2}$. For this reason we will consider double covers of the groups in question. For a subgroup $\Gamma$ of $S O(3), \tilde{\Gamma}$ will denote its inverse image in the usual double covering $S U(2) \rightarrow S O(3)$. Then a finite subgroup of $S U(2)$ is of the form $\tilde{\Gamma}$ for some $\Gamma \subseteq S O(3)$ if and only if $\Gamma$ is not cyclic of odd order. From our discussion above we now have the following corollary.

COROLlARY 3.1. There is a bijection $\mu$ between the set of isomorphism classes of complex simple Lie algebras of type A,D and $E$ and the isomorphism classes of non-trivial finite subgroups of $S U(2)$ namely:

(i) $\mu\left(A_{2 n-1}\right)=\tilde{C}_{n}$;

(ii) $\mu\left(A_{2 n}\right)=C_{2 n-1}$;

(iii) $\mu\left(D_{n+2}\right)=\tilde{D}_{2 n}$; 
(iv) $\mu\left(E_{6}\right)=\tilde{A}_{4}$;

(v) $\mu\left(E_{7}\right)=\tilde{S}_{4}$;

(vi) $\mu\left(E_{8}\right)=\tilde{A}_{5}$.

We now prove an analogue of the above theorem for the Dynkin diagram of certain irreducible representations of $\mathbf{g}$.

THEOREM 3.2. Let $\triangle_{\omega_{j}}$ be the Dynkin diagram of an irreducible representation of $\mathbf{g}$ (of type $A, D$ orE) with highest weight $\omega_{j}$, where $\alpha_{j}$ is a pendant vertex of $\triangle_{\mathbf{g}}$, and let $\alpha_{s}$ be a node of $\triangle_{\mathbf{g}}$ with maximal degree $k$ and distances $v_{1}-1, \ldots, v_{k}-1$ from the corresponding pendant vertices, $v_{*}-1$ denoting the distance to $\alpha_{j}$, Then $d_{s}^{j}$ satisfies

$$
\sum_{i=1}^{k}\left(1-\frac{1}{v_{i}}\right)=2-\frac{2}{2 d_{s}^{j} v_{*}} .
$$

In particular for the corresponding finite subgroup of $S U(2)$ we have that $|\tilde{\Gamma}|=4 d_{s}^{j} v_{*}$, where again if $\mathbf{g}$ is of type $A$, it is assumed to be of odd rank and $\alpha_{s}$ is the middle vertex.

Proof. From Proposition 2.1 we have that the vertex coefficients $d_{i}^{j}$ of any simple path in $\tilde{\triangle}_{\omega_{j}}$, starting at a pendant vertex and ending at the first vertex with a branch or multiple connection, form an arithmetic progression. In particular for such a path in $\triangle_{\omega_{j}}$ not containing $\alpha_{j}$ we have that

$$
d_{i_{v_{l}-1}}^{j}=\frac{v_{l}-1}{v_{l}} d_{s}^{j}
$$

whereas in the branch containing $\alpha_{j}$

$$
d_{i_{v-1}}^{j}=\frac{\nu_{l}-1}{v_{l}} d_{s}^{j}+1-\frac{\nu_{*}-1}{v_{*}} .
$$

Summing these equations over the branches we obtain

$$
\sum_{l=1}^{k} d_{i_{v-1}}^{j}=d_{s}^{j}\left(\sum_{l=1}^{k} \frac{v_{l}-1}{v_{l}}\right)-\frac{v_{*}-1}{v_{*}} .
$$

Subtracting the additional equation $\sum_{l=1}^{k} d_{i_{v_{l}-1}}^{j}=2 d_{s}^{j}+\delta_{j s}=2 d_{s}^{j}$, (since a pendant vertex is never of maximal degree), we obtain

$$
\left(\sum_{l=1}^{k}\left(1-\frac{1}{v_{l}}\right)-2\right) d_{s}^{j}=-\frac{1}{v_{*}},
$$

and hence $\sum_{i=1}^{k}\left(1-\frac{1}{v_{i}}\right)=2-\frac{2}{2 d_{s}^{j} \nu_{*}}$.

4. Plücker formulae. In this section we relate our results to the generalised Plücker formulae obtained in [1]. We first recall the context and notation there. Given a compact simple Lie group $G$ with maximal torus $T$, let $\mathfrak{g}^{\mathbb{C}}=\mathfrak{t}^{\mathbb{C}} \oplus \bigoplus_{\alpha \in \Delta} \mathfrak{g}^{\alpha}$ be the corresponding root space decomposition of the complexification of $\mathfrak{g}$. Identifying $\mathfrak{g} \cap \bigoplus_{\alpha \in \Delta} \mathfrak{g}^{\alpha}$ with the tangent space at the identity coset of $G / T$ via the derivative (at the identity element of G) of the canonical projection $\pi: G: \rightarrow G / T$ yields the 
$G$-invariant decomposition $T^{\complement} G / T=\bigoplus_{\alpha \in \Delta}\left[\mathfrak{g}^{\alpha}\right]$, where $\mathfrak{g}^{\alpha}$ is the fibre of $\left[\mathfrak{g}^{\alpha}\right]$ at the identity coset.

A smooth map $\psi: S: \rightarrow G / T$ of a compact Riemann surface $S$ into the full flag manifold $G / T$ is said to be $\tau$-adapted if $d \psi\left(T^{1,0} S\right) \subseteq \bigoplus_{j=0}^{r}\left[\mathfrak{g}^{\alpha_{j}}\right]$, where $\left\{\alpha_{1}, \ldots \alpha_{r}\right\}$ are a choice of simple roots with respect to $\operatorname{tand}-\alpha_{0}=m_{1} \alpha_{1}+\cdots+m_{r} \alpha_{r}$ is the highest root. There is a canonical connection on $G / T$ and given a $\tau$-adapted map $\psi: S: \rightarrow G / T$ the induced connection $D^{\alpha}$ on the pull-back bundle $\psi^{*}\left[\mathfrak{g}^{\alpha}\right]$ endows it with the structure of a holomorphic line bundle. For $j=o, \ldots, r$ let $\partial^{[j]}: T^{1,0} S \rightarrow \psi^{*}\left[\mathfrak{g}^{\alpha}\right]$ be the homomorphism of complex line bundles defined by $\partial^{[j]}\left(\frac{\partial}{\partial z}\right)=\left(d \psi\left(\frac{\partial}{\partial z}\right)\right)^{\left[\mathfrak{g}^{\alpha_{j}}\right]}$, where $(X)^{\left[\mathfrak{g}^{\alpha_{j}}\right]}$ is the $\left[\mathfrak{g}^{\alpha_{j}}\right]$ component of $X \in T^{\mathbb{C}} G / T$. The homomorphisms $\partial^{[j]}$ are holomorphic when $\psi$ is $\tau$-adapted and so have isolated zeros if they are not identically zero. When $\partial^{[0]}$ is not identically zero $\psi$ is said to be $\tau$-primitive, otherwise $\psi$ is said to be $\tau$-holomorphic, as this condition is equivalent to $\psi$ being holomorphic. When $\partial^{[j]}$ is not identically zero for $j=1, \ldots, r$ a $\tau$-adapted map is said to be $\partial$-full. For example in the classical case when $G=S U(n+1)$ and $\psi$ is the Frenet frame of a linearly full holomorphic curve in $\mathbb{C} P^{n}, \psi$ is $\partial$-full and $\tau$-holomorphic. The classical associated curves of such a linearly full holomorphic curve generalise to the associated curves of a $\tau$-adapted map $\psi: S: \rightarrow G / T$ as follows. Let $P_{j}$ be the maximal parabolic subgroup with Lie algebra $\mathfrak{t}^{\mathbb{C}} \oplus \bigoplus_{\alpha \in \Delta \backslash \Pi_{j}} \mathfrak{g}^{\alpha}$, where $\Pi_{j}$ is the set of positive roots with positive $\alpha_{j}$ coefficient when expressed in terms of the simple roots. Setting $K_{j}=G \cap P_{j}$, so that $G / K_{j}=G^{\mathbb{C}} / P_{j}$ is a complex manifold, we obtain a natural holomorphic projection $\pi_{j}: G / T \rightarrow G / K_{j}$. If $V_{j}$ is the $j$ th fundamental representation of $G^{\mathbb{C}}$, and $v_{j} \in V_{j}$ is a lowest weight vector with weight $-\omega_{j}$, then $P_{j}$ is the stabiliser of $\left[v_{j}\right] \in \mathbb{P}\left(V_{j}\right)$, and the inclusion $i_{j}: G / K_{j}=G^{\mathbb{C}} / P_{j} \subset \mathbb{P}\left(V_{j}\right)$ of $G^{\mathbb{C}} / P_{j}$ as the orbit of $\left[v_{j}\right]$ is holomorphic. The $j$ th associated curve is then given by $\tilde{\psi}_{j}=i_{j} \pi_{j} \psi: S \rightarrow \mathbb{P}\left(V_{j}\right)$, and the pullback of the tautological line bundle over $\mathbb{P}\left(V_{j}\right)$ by $\tilde{\psi}_{j}$ gives a corresponding line bundle $\mathcal{L}_{j}$. When $V_{j}$ is equipped with a $G$-invariant Hermitian inner product we obtain a connection $D^{\mathcal{L}_{j}}$ on $\mathcal{L}_{j}$ by taking $D_{X}^{\mathcal{L}_{j}}$ to be the $\mathcal{L}_{j}$ component of $X s$ in the orthogonal decomposition $V_{j}=\mathcal{L}_{j} \oplus \mathcal{L}_{j}^{+}$, where $s: S \rightarrow V_{j}$ is a section of $\mathcal{L}_{j}$. Integrating the Chern form of this connection over $S$ one obtains the Chern number $c_{1}\left(\mathcal{L}_{j}\right)$ of the bundle, and the degree $\delta_{j}$ of the associated curve $\tilde{\psi}_{j}$ is therefore given by $\delta_{j}=-c_{1}\left(\mathcal{L}_{j}\right)$. Finally the ramification indices $r^{j}$ of the bundle maps $\partial^{[j]}$ are defined to be the sum of the orders of their zeros. We can now state the generalisation of the Plücker formulae obtained in [1].

THEOREM 4.1. (See [1]). Let $\psi: S: \rightarrow G / T$ be a $\partial$-full $\tau$-adapted map of a compact Riemann surface $S$ with Euler characteristic $\chi(S)$ into the full flag manifold $G / T$ of a compact simple Lie group $G$. Then

$$
r^{j}+\chi(S)=\sum_{k=1}^{r} c_{j k} \delta_{k},
$$

where $\left(c_{j k}\right)$ is the extended Cartan matrix of the Lie algebra of $G, j=1, \ldots, r$ if $\psi$ is $\tau$-holomorphic and $j=0, \ldots, r$ if $\psi$ is $\tau$-primitive.

A modification of the arguments of Lemma 3.1 and Theorem 3.1 gives the following, where $R=\sum_{j=1}^{k} \frac{R_{j}}{v_{j}}$, and $R_{j}$ denotes the sum of the ramification indices in the $j$-th branch of the Dynkin diagram weighted by their positions in the branch 
(the branch node may be counted in any, but only one branch), and $\tilde{\Gamma}$ is the finite subgroup of $S U(2)$ corresponding to $\mathbf{g}$ of type $A$ (of odd rank), $D$ or $E$.

THEOREM 4.2. Let $\triangle_{\mathbf{g}}$ be the Dynkin diagram of a simply laced complex simple Lie algebra of rank $r$, and let $\alpha_{s}$ be a node of $\triangle_{\mathbf{g}}$ with maximal degree $k$ and distances $v_{1}-1, \ldots, v_{k}-1$ from the corresponding pendant vertices. If $\psi: S: \rightarrow G / T$ is a $\partial$-full $\tau$-adapted map of a compact Riemann surface $S$ with Euler characteristic $\chi(S)$ into the corresponding full flag manifold $G / T$, then

$$
\left[\sum_{j=1}^{k}\left(1-\frac{1}{v_{j}}\right)-2\right] \delta_{s}=-\frac{\chi(S)(r+1)+2 R}{2} .
$$

COROLlary 4.1. Let $\psi$ be $\partial$-full and $\tau$-holomorphic:

(i) $\frac{4 \delta_{s}}{\chi(S)(r+1)+2 R}=\frac{2 a_{s}}{r+1}=|\Gamma|$.

(ii) (See [1].) If $r^{j}=0$ for $j=1, \ldots, r$, then $\delta_{j}=a_{j}$ for $j=1, \ldots, r$, and $\chi(S)=2$.

(iii) If $\chi(S)=-c, c \geq 0$, then $2 R>c(r+1)$.

Let $\psi$ be $\partial$-full and $\tau$-primitive.

(iv) (See [1].) $\sum_{j=0}^{r} m_{j} r^{j}=-\chi(S) h$. Here $h$ is the Coxeter number of $G$.

(v) For the root systems $D_{r}, r \neq 4$ and $E_{7}$, there are no $\partial$-full $\tau$-primitive maps with $r^{j}=m_{j}$, for $j=0, \ldots, r$.

Proof. (i) is an immediate consequence of Theorem 3.1 and Theorem 4.2. To prove (ii) we note that if $r^{j}=0$ for $j=1, \ldots, r$, then $R=0$, so that by (i) $4 \delta_{s}=2 \chi(S) a_{s}$, and since $\delta_{s}$ is positive (as the degree of a holomorphic map), $\chi(S)>0$, and so $\chi(S)=2$ and $\delta_{s}=a_{s}$. This and the fact that the $\delta_{j}$ 's and the $a_{j}$ 's satisfy the same recurrence relations gives the required equality for all $j$. The proof of (iii) again follows from Theorem 4.2, the fact that $\delta_{s}$ is positive and that $\sum_{j=1}^{k}\left(1-\frac{1}{v_{j}}\right)<2$.

When $\psi$ is $\partial$-full and $\tau$-primitive the recurrence relations must be solved in the extended Dynkin diagram so that (with distances now measured in the extended diagram) $\sum_{j=1}^{k}\left(1-\frac{1}{v_{j}}\right)-2=0$, (the case of $A_{r}$ must be dealt with separately but similarly), and the corresponding right hand side of the equation in Theorem 4.2 for the extended diagram must therefore also be zero. Using this and the fact that $\sum_{j=0}^{r} m_{j}=h$ proves (iv). Finally if $r^{j}=m_{j}$ for $j=0, \ldots, r$. then by (iv) $\sum_{j=0}^{r} m_{j}^{2}=-\chi(S) h$. However by the McKay correspondence we know that the $m_{j}$ 's are equal to the degrees of the irreducible characters of the corresponding $\tilde{\Gamma}$, so that $\sum_{j=0}^{r} m_{j}^{2}=|\tilde{\Gamma}|$. For the root systems $D_{r}, r \neq 4$ and $E_{7}$, the equality $|\tilde{\Gamma}|=-\chi(S) h$ violates the integrality of $\chi(S)$.

\section{REFERENCES}

1. J. Bolton and L. M. Woodward, Toda equations and Plücker formulae, Bull. London Math. Soc. 35, (2003), 145-151.

2. N. Bourbaki, Groupes et algèbres de Lie. Ch. 4, 5 et 6 (Hermann, Paris, 1968).

3. H. M. Farkas and I. Kra, Riemann surfaces (Springer-Verlag, 1980). 1972).

4. J. E. Humphreys. Introduction to Lie algebras and representation theory (Springer-Verlag,

5. J. A. Wolf. Spaces of constant curvature (McGraw-Hill, New York, 1967). 